\title{
anatomy
}

Original Article

http://dergipark.org.tr/en/pub/anatomy

Received: November 13, 2020; Accepted: May 19, 2021

doi:10.2399/ana.21.825667

\section{Variations of the branches arising from the third part of the axillary artery: a cadaveric study}

\author{
Abduelmenem Alashkham $^{1-3}$ (D), Tarek Almabrouk ${ }^{3,4}$ (D), Roger Soames ${ }^{2}$ (D) \\ ${ }^{1}$ Anatomy, School of Biomedical Sciences, University of Edinburgh, Edinburgh, UK \\ ${ }^{2}$ Centre for Anatomy and Human Identification, University of Dundee, Dundee, UK \\ ${ }^{3}$ Human Anatomy Department, Faculty of Medicine, University of Zawia, Zawia, Libya \\ ${ }^{4}$ St. Georges International School of Medicine, Northumbria University, Newcastle Upon Tyne, UK
}

\begin{abstract}
Objectives: This study aims to describe variations of the third part of the axillary artery branches with respect to their diameter, origin, subsequent branches, and gender.

Methods: One-hundred and forty cadaveric shoulders were examined bilaterally in the Centre for Anatomy and Human Identification, University of Dundee, which is regulated by Human Tissue Act (Scotland) 2006. Branches arising from the third part of the axillary artery were dissected and the diameter, origin, subsequent branching patterns of each branch were documented, as well as gender. T-tests and chi-square tests were used to determine the association between the variables.

Results: The most common variations were associated with the posterior circumflex humeral artery (48.3\%), followed by the anterior circumflex humeral (25\%) and subscapular arteries (16.7\%). Variations of the anterior circumflex humeral artery were more common in females than males $(p<0.05)$. A significant association between the origin of posterior circumflex humeral artery and its subsequent branching pattern was observed. In females, the anterior circumflex humeral artery was more likely to give rise to the profunda brachii when it originated from the posterior circumflex humeral artery.

Conclusion: Branches of the third part of the axillary artery vary in diameter, origin and subsequent branching patterns and the gender. An understanding of these variations is essential for accurate radiological diagnostic interpretation and therapeutic intervention. The difference in the variations observed and those reported in the literature suggest the need for further large scale studies.
\end{abstract}

Keywords: axilla; axillary artery; shoulder; variations

Anatomy 2021;15(2):104-115 @2021 Turkish Society of Anatomy and Clinical Anatomy (TSACA)

\section{Introduction}

The axillary artery is a continuation of the subclavian artery at the outer border of the first rib. It continues as the brachial artery at the lower border of teres major. Pectoralis minor lies anterior to the artery dividing it into three parts: the first part (suprapectoral) is proximal between the lateral border of the first rib and medial border of pectoralis minor; the second part (retropectoral) lies posterior to it; and the third part (infrapectoral) extends from the lateral border of pectoralis minor to the lower border of teres major. During its course the axillary artery gives six branches: the superior thoracic artery from the first part; the thoracoacromial and lateral thoracic arteries from the second part; and the anterior and posterior circumflex humeral and subscapular arteries from the third part. Its continuation, the brachial artery, gives the profunda brachii artery. ${ }^{[1-3]}$

The vasculature of the upper limbs originates from the primitive axial and superficial brachial arteries, with the axillary, brachial and interosseous arteries arising from the primitive axial artery. In the proximal arm, both the brachial and axillary arteries merge with the superficial

The abstract has been presented at the Summer Meeting of the British Association of Clinical Anatomists UCLan School of Medicine, Greenbank Lecture Theater, Greenbank Building, University of Central Lancashire, 2 7uly 2019, Preston, UK. 
brachial artery, while in its distal part, the superficial brachial artery anastomoses with the brachial artery. In the forearm, the primitive axial artery (ulnar system) gives a terminal trunk which anastomoses with the median artery (derivative of forearm arteries), the deep branch of the radial artery (branch of the primitive axial artery) and the ulnar artery. ${ }^{[4,5]}$ Differentiation of the vessels occurs proximal to distal by regression of some capillaries and maintenance and enlargement of others. It is the variations in differentiation, regression and persistence of these capillaries which result in the variations seen in the vessels of the upper limb..$^{[5]}$ An in-depth knowledge of the normal arterial anatomy of the axillary artery and its variants is essential for clinical diagnosis and treatment, for example in coronary bypass and shoulder dislocation. ${ }^{[6]}$

Nevertheless, a series of case studies report variations in the branching pattern of the 3rd part of the axillary artery: variations in origin, ${ }^{[7,8]}$ course , $^{[9,10]}$ the presence of common trunks, ${ }^{[11-13]}$ and of additional branches, such as the radial artery. ${ }^{[14]}$ According to Rao et al. ${ }^{[1]]}$ and Olinger ${ }^{[15]}$ the classical description of the axillary artery is only present in $10 \%$ of cases. The most common variation is a common trunk rather than individual branches, followed by branches arising distally or from proximal branches, such as the deep brachial artery arising from the posterior circumflex humeral artery, which according to Olinger ${ }^{[15]}$ occurs in $5 \%$ of cases.

Variations of the third part of the axillary artery have been reported either as scattered in the literature, ${ }^{[6,11,15-17]}$ or reviews on a small number of cadavers. ${ }^{[18-20]}$ The subscapular artery, largest branch of the 3rd part of the axillary artery, arises from a common trunk with the posterior circumflex humeral artery in up to $30 \%$ of cases. Furthermore, either the subscapular, anterior or posterior circumflex humeral arteries, as well as the profunda brachii having been reported arising from a common trunk. ${ }^{[21]}$ One cadaveric study using 423 upper limbs reported truncus subscapulocircumflexus in $22.9 \%$, truncus profundocircumflexus in $13.75 \%$, and truncus bicircumflexus in $13.95 \%$ of the cases. ${ }^{[21]}$

Consequently, an inconsistency exists in the literature in which variations of the 3rd part of the axillary artery may be presented as an exception or may occur in high frequencies. For example, Miguel-Perez ${ }^{[2]}$ and Siri et al. ${ }^{[23]}$ found only one variation among 36 and 50 upper limbs respectively. ${ }^{[21-23]}$ Others, however, report variations with greater frequency, with Gaur et al. ${ }^{[2]}$ observing variations in $16 \%$ of 50 specimens and Maheswary Thampi et al. ${ }^{[20]}$ reporting variations in $40 \%$ of 40 shoulders examined. It is difficult, therefore to determine the incidence of variations. Furthermore, many studies tend to focus on the frequencies of the variations and do not provide sufficient information on their relationship to gender, for example. ${ }^{[9,19,22]}$

A knowledge of variations in the branching pattern of the axillary artery is important for both diagnostic purposes and therapeutic interventions. ${ }^{[25-31]}$ Vascular radiologists acknowledge the significance of variations when conducting angiographic imaging ${ }^{[6]}$ while surgeons may be faced with interpreting arterial variations in procedures for trauma or neoplasm. ${ }^{[2]]}$ The current study, therefore, aims to determine the incidence of variations in the branching pattern of the 3rd part of the axillary artery, and describe these variations with respect to their association with vessel diameter, side, and gender.

\section{Materials and Methods}

A total of 140 cadaveric shoulders from 70 cadavers of British origin (30 males and 40 females), with an average age of 81.5 (range 53-101) years were dissected and examined. The cadavers had been donated to the Centre for Anatomy and the Human Identification University of Dundee: all donations are in line with the Human Tissue Act (Scotland) 2006. Each shoulder was dissected following classical incisions and dissection procedures described in Grant's Dissector 16th edition. ${ }^{[32]}$ Careful dissection was carried out to expose the axillary region, following which the $3 \mathrm{rd}$ part of the axillary artery and its branches were inspected: the presence of additional was also documented. The origin of each branch or common trunk was noted, as was its relation to the axillary artery, i.e. from which aspect it arose. The diameter (in $\mathrm{mm}$ ) of each artery/common trunk at its origin was taken.

Classical descriptions of the 3 rd part of the axillary artery state that it has three single branches, the anterior and posterior circumflex humeral, and subscapular arteries. Deviations from this pattern were considered as being a variation.

The data were entered into with Statistical Package for Social Sciences (SPSS Version 20, Armonk; NY, USA) for subsequent analysis. In the results the incidence of variations is presented. This is followed by a consideration of the variations in origin on the right and left sides, the branches observed, and the diameter or common origin of the anterior and posterior circumflex humeral, and subscapular arteries are described. Categorical variables are presented as frequencies and percentages of occurrence. Vessel diameter, as a continuous variable, was tested for its distribution using the Kolmogorov-Smirnov test: diameter is described by its mean and associated standard deviation. Inferential statistics were carried out to determine the presence of statistical differences when comparing characteristics of the arteries inspected with demographic vari- 
ables. Independent t-tests, chi-square tests and one-way ANOVA were conducted. Chi-square post-hoc analysis with Bonferroni adjustment was carried out if statistically significant differences were observed. Correlation analysis, using the Pearson coefficient, was conducted between vessel diameter and age. All results were considered significant if $\mathrm{p}$-value was less than 0.05 .

\section{Results}

Variations in the 3rd part of the axillary artery were observed in $80 \%$ of specimens, with only $20 \%$ showing the classical anatomical pattern bilaterally. Notably, 95 specimens $(67.9 \%)$ showed variations of which $52(54.7 \%)$ were on the right and 43 (45.3\%) the left side (Table 1). No significant differences was observed between right and left $\operatorname{limbs}\left(\chi^{2}=2.653, \mathrm{p}>0.05\right)$. Comparison of the incidence of variations in males and females revealed $36.8 \%(n=35)$ of variations were observed in males, and $63.2 \%(\mathrm{n}=60)$ in females (Table 1). There was a significant difference

\section{Table 1}

The number of 'classical' origin of branches from the third part of the axillary artery, together with the percentages of the variations observed in the right and left limbs, as well as between genders.

\begin{tabular}{lccccc} 
& \multicolumn{2}{c}{ Limb } & & \multicolumn{2}{c}{ Gender } \\
\cline { 2 - 3 } \cline { 6 - 6 } & Left & Right & & Male & Female \\
\hline Classical origin & 27 & 18 & 25 & 20 \\
\hline Variation & $43(45.3 \%)$ & $52(54.7 \%)$ & $35(36.8 \%)$ & $60(36.8 \%)$ \\
\hline Total & 70 & 70 & 60 & 80 \\
\hline
\end{tabular}

between males and females $\left(\chi^{2}=4.366, \mathrm{p}<0.05\right)$. No difference was observed between the mean age of specimens with and without variation $(\mathrm{p}>0.05)$.

Variation in the origin of the anterior circumflex humeral artery was observed in 58 specimens (41.4\%) (Figures 1-3). It most frequently arose from the 3rd part of the axillary artery $(85.7 \%, \mathrm{n}=106)$, the posterior circum-

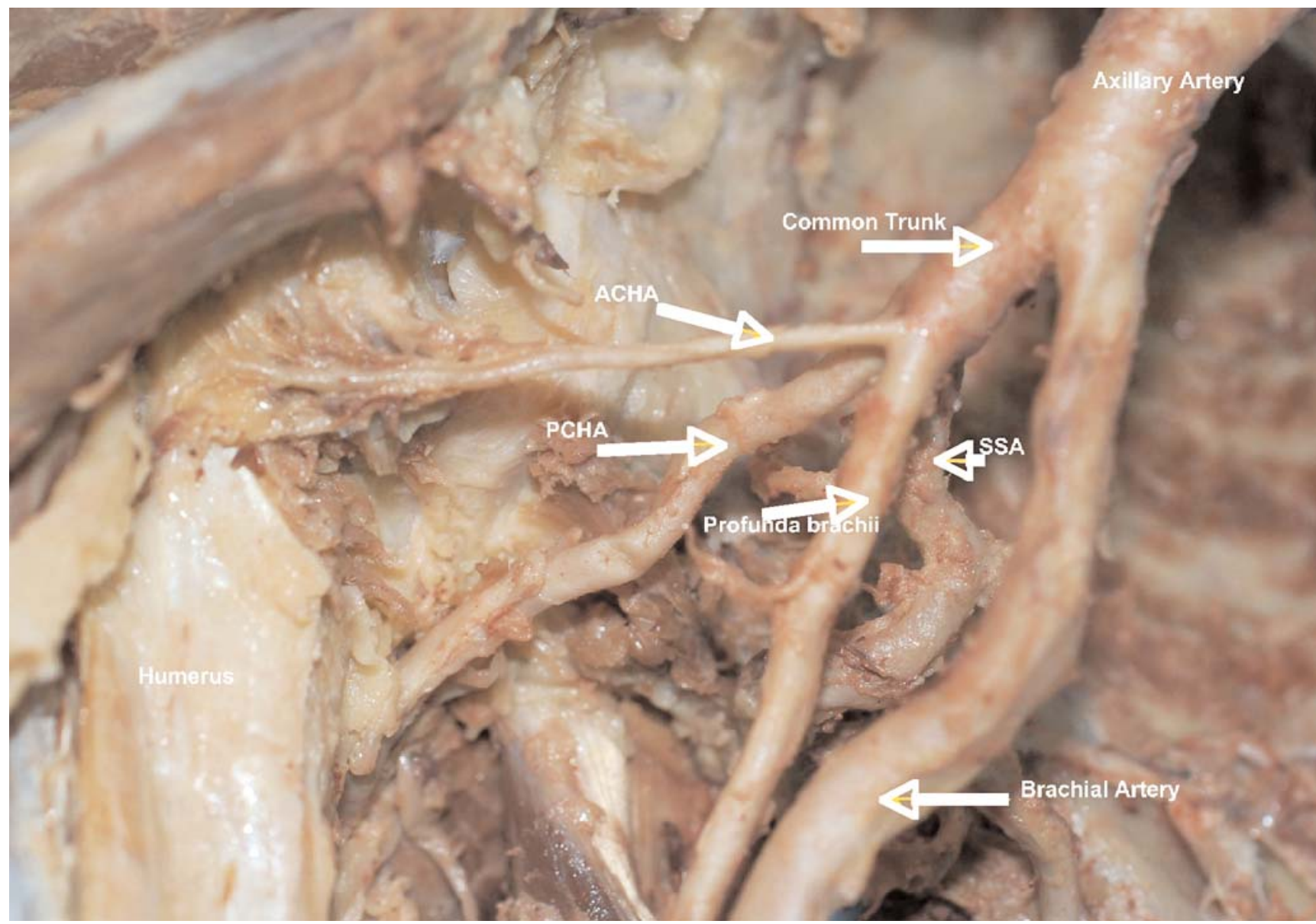

Figure 1. Anterolateral view of the right shoulder showing the axillary artery giving a common trunk, which divides into the subscapular artery (SSA), the posterior circumflex humeral artery (PCHA), the anterior circumflex humeral artery (ACHA) and profunda brachii. 


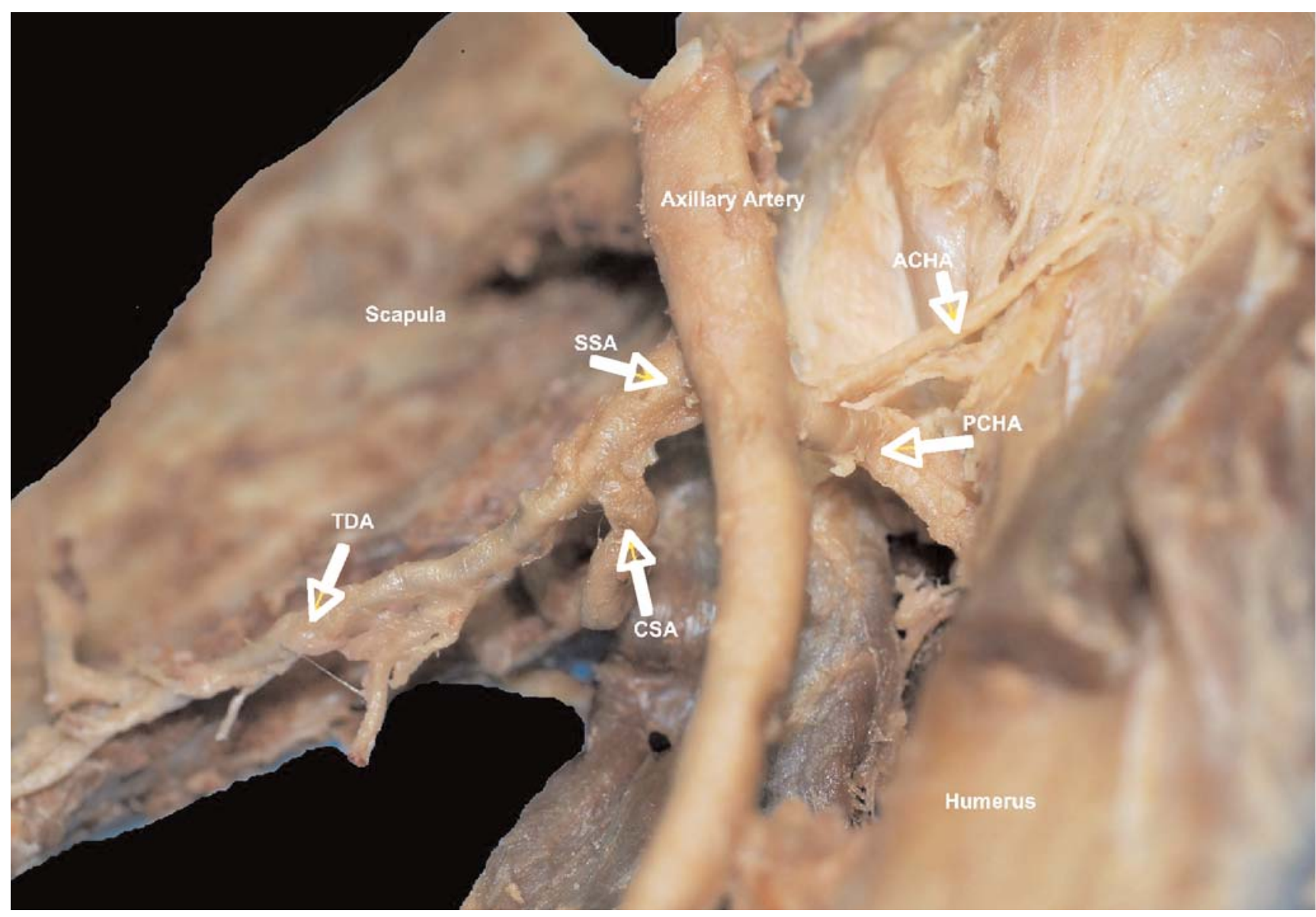

Figure 2. Anterior aspect of the left shoulder showing the third part of the axillary artery (AA) and its branches, the subscapular artery (SSA) and the anterior (ACHA) and posterior circumflex humeral arteries (PCHA). Note the ACHA and PCHA arise from a common trunk. CSA: circumflex scapular artery; TD: thoracodorsal artery.

flex humeral artery in 15 specimens (11.4\%), profunda brachii in 3 specimens $(2.1 \%)$ and the 1 st part of the axillary artery in 1 specimen $(0.7 \%)$ (Table 2$)$. In 129 speci-

\section{Table 2}

Origin of the anterior circumflex humeral artery, posterior circumflex humeral artery and the subscapular artery.

\begin{tabular}{lccc} 
Origin & ACHA & PCHA & SSA \\
\hline 3rd part AA & $120(85.7 \%)$ & $106(75.7 \%)$ & $127(90.7 \%)$ \\
\hline PCHA & $15(10.7 \%)$ & - & - \\
\hline PB & $4(2.9 \%)$ & $4(2.9 \%)$ & $1(0.7 \%)$ \\
\hline 1st part AA & $1(0.7 \%)$ & - & - \\
\hline BA & - & $20(14.2 \%)$ & - \\
\hline SSA & - & $9(6.4 \%)$ & - \\
\hline CSA & - & $1(0.7 \%)$ & - \\
\hline 2nd part AA & - & - & $12(8.5 \%)$ \\
\hline Total & $140(100 \%)$ & $140(100 \%)$ & $140(100 \%)$ \\
\hline
\end{tabular}

AA: axillary artery; ACHA: anterior circumflex humeral artery; BA: brachial artery; CSA: circumflex scapular artery; PCHA: posterior circumflex humeral artery; SSA: subscapular artery. mens $(92.1 \%)$ the artery arose as a single branch, while in 11 specimens $(7.9 \%)$ it had a common origin with the posterior circumflex humeral artery (Table 3; Figure 2).

In 99 specimens the anterior circumflex humeral artery originated from the lateral side $(70.7 \%)$ of the axillary artery. The anterior circumflex humeral artery originated posterolaterally in 25 specimens $(17.9 \%)$, superiorly in $8(5.7 \%)$, anterolaterally in $4(2.9 \%)$, and from the posterior, anterior, anterosuperior and posterosuperior aspects in 1 specimen each $(0.7 \%)$ (Table 4). In 11 specimens both anterior and posterior circumflex humeral arteries arose from a common trunk, while in 21 specimens $(15 \%)$ the anterior circumflex artery gave the profunda brachii as a branch.

Variations were observed in 21 male and 37 female specimens, with a significant difference between origin and gender being observed $(\mathrm{p}<0.05)$. Further analysis, using post-hoc tests, showed significant differences in the aspect of origin from the 3 rd part of the axillary artery $(\mathrm{p}<0.05)$ 


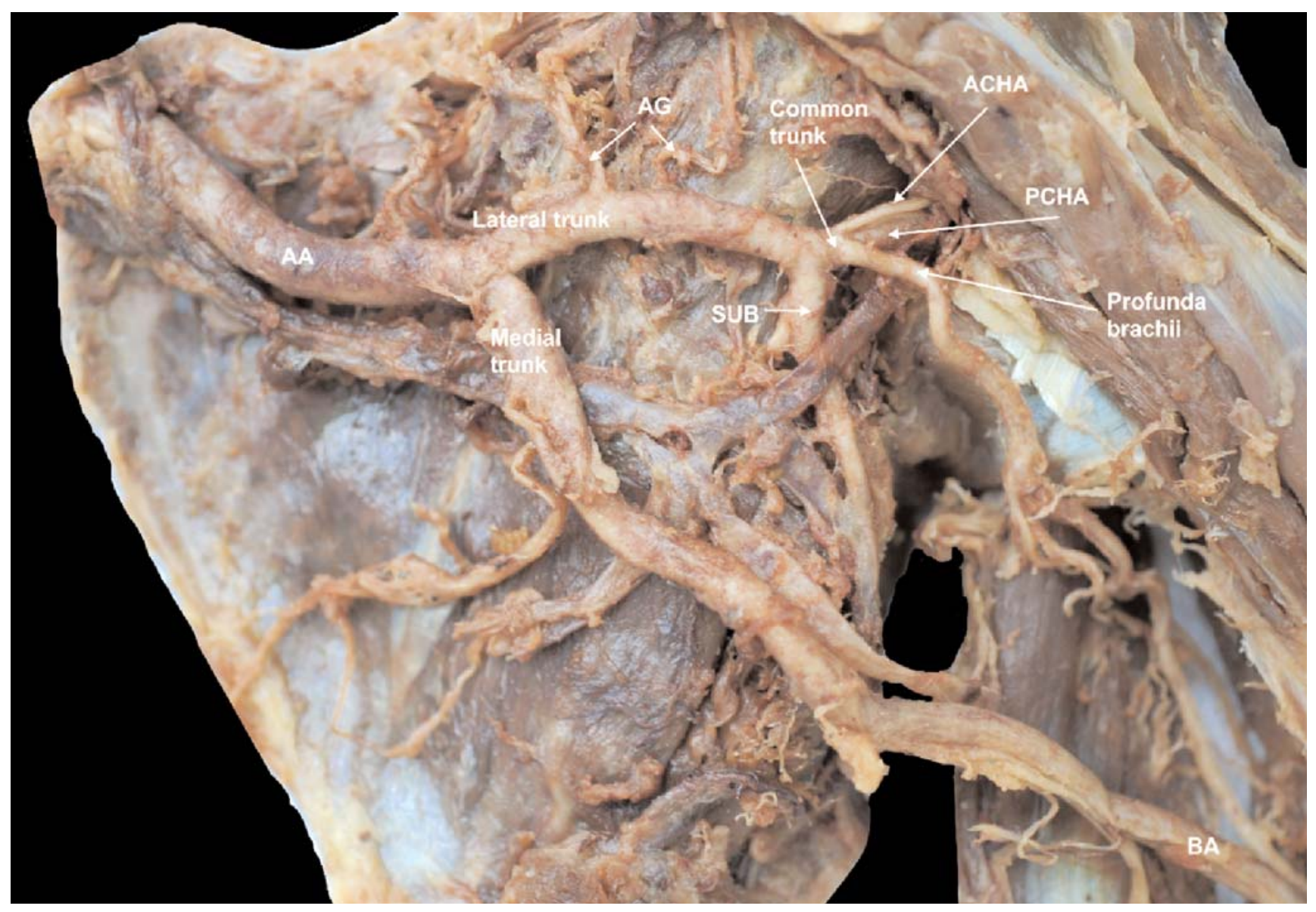

Figure 3. Anterior view of the left shoulder showing the axillary artery dividing into lateral and medial trunks. The lateral trunk gives a common trunk, which divides into anterior circumflex humeral $(\mathrm{ACHA})$, posterior circumflex humeral $(\mathrm{PCHA})$ and profunda brachii $(\mathrm{PB})$ arteries. The medial trunk becomes the brachial artery (BA).

(Table 3). The mean diameter of the anterior circumflex humeral artery was $2.15 \pm 0.06 \mathrm{~mm}$, with no difference being observed between males and females $(\mathrm{p}>0.05)$.
However, a significant difference was observed in diameter depending on its aspect of origin $(\mathrm{p}<0.05)$. Significant differences were also revealed when comparing the diameter

Table 3

The frequency with which the anterior circumflex humeral artery, posterior circumflex humeral artery and the subscapular artery arose from a common trunk with other branches.

\begin{tabular}{|c|c|c|c|c|}
\hline Origin & ACHA & PCHA & SSA & Total \\
\hline as a single branch & $129(92.1 \%)$ & $91(65 \%)$ & $109(77.9 \%)$ & $329(78 \%)$ \\
\hline with PCHA & 11 (7.9\%) & - & 27 (19.3\%) & 38 (9\%) \\
\hline with ACHA & - & $11(7.9 \%)$ & $1(0.7 \%)$ & $12(2.9 \%)$ \\
\hline with $\mathrm{ACHA}$ and $\mathrm{PCHA}$ & - & - & $1(0.7 \%)$ & $1(0.2 \%)$ \\
\hline with PCHA and CSA & - & - & $1(0.7 \%)$ & $1(0.2 \%)$ \\
\hline with $\mathrm{ACHA}$ and $\mathrm{PB}$ & - & - & $1(0.7 \%)$ & $1(0.2 \%)$ \\
\hline with SSA & - & $30(21.4 \%)$ & - & $30(7.1 \%)$ \\
\hline with PB & - & $7(5 \%)$ & - & $7(1.7 \%)$ \\
\hline with CSA & - & $1(0.7 \%)$ & - & $1(0.7 \%)$ \\
\hline
\end{tabular}

ACHA: anterior circumflex humeral artery; CSA: circumflex scapular artery; PB: profunda brachii; PCHA: posterior circumflex humeral artery; SSA: subscapular artery. 
Table 4

The aspect of the third part of the axillary artery from which the anterior circumflex humeral artery, posterior circumflex humeral artery and the subscapular artery originated.

\begin{tabular}{lcccc} 
Site of origin & ACHA & PCHA & SSA & Total \\
\hline Lateral & $99(70.7 \%)$ & $27(19.3 \%)$ & - & $126(30 \%)$ \\
\hline Postero-lateral & $25(17.9 \%)$ & $46(32.9 \%)$ & - & $71(16.9 \%)$ \\
\hline Antero-lateral & $4(2.9 \%)$ & - & - & $4(1 \%)$ \\
\hline Superior & $8(5.7 \%)$ & $3(2.1 \%)$ & - & $11(2.6 \%)$ \\
\hline Posterior & $1(0.7 \%)$ & $63(45 \%)$ & - & $71(16.9 \%)$ \\
\hline Anterior & $1(0.7 \%)$ & - & - & $1(0.2 \%)$ \\
\hline Antero-superior & $1(0.7 \%)$ & - & - & $1(0.2 \%)$ \\
\hline Postero-superior & $1(0.7 \%)$ & - & $97(69.3 \%)$ & $1(0.2 \%)$ \\
\hline Medial & - & $1(0.7 \%)$ & $24(17.1 \%)$ & $97(23.1 \%)$ \\
\hline Posteromedial & - & - & $12(8.6 \%)$ & $25(6 \%)$ \\
\hline Inferior & - & - & - & $12(2.9 \%)$ \\
\hline
\end{tabular}

ACHA: anterior circumflex humeral artery; PCHA: posterior circumflex humeral artery; SSA: subscapular artery.

between age groups, but there was no correlation between vessel diameter and age. No differences were found in diameter irrespective of whether the artery arose from a common origin or the aspect from which it arose, or relation to side, age or gender.
Variation in the origin of the posterior circumflex humeral artery was reported in 112 specimens (80\%). The most common origin for the posterior circumflex humeral artery (Figures 1-6) was from the 3rd part of the axillary artery $(106,75.7 \%)$, the brachial artery in 20 speci-

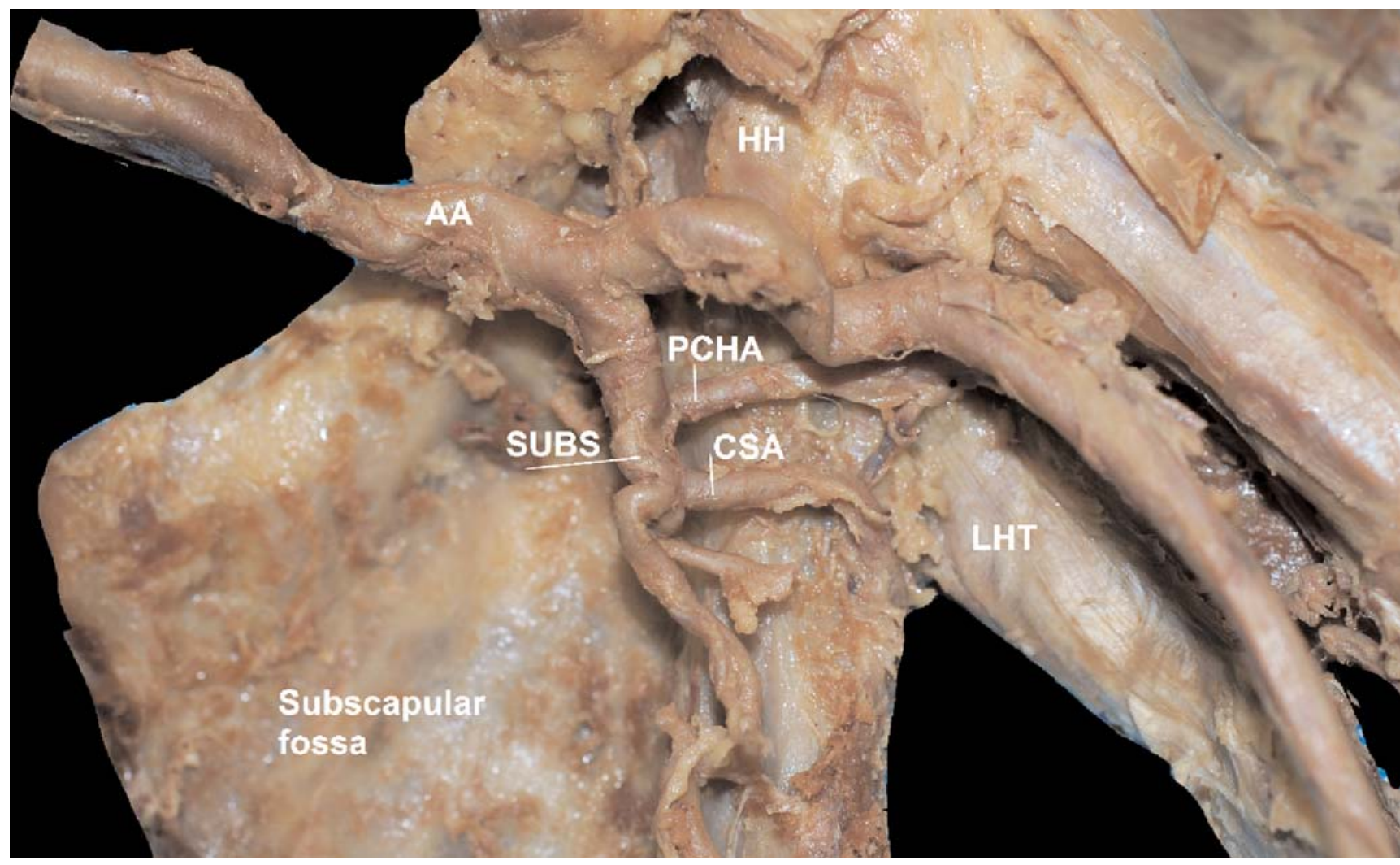

Figure 4. Anterior view of the left shoulder showing dissection of the inferior aspect of the glenohumeral joint. AA: axillary artery; CSA: circumflex scapular artery; HH: humeral head; LHT: long head of triceps; PCHA: posterior circumflex humeral artery; SUBS: subscapular artery. 


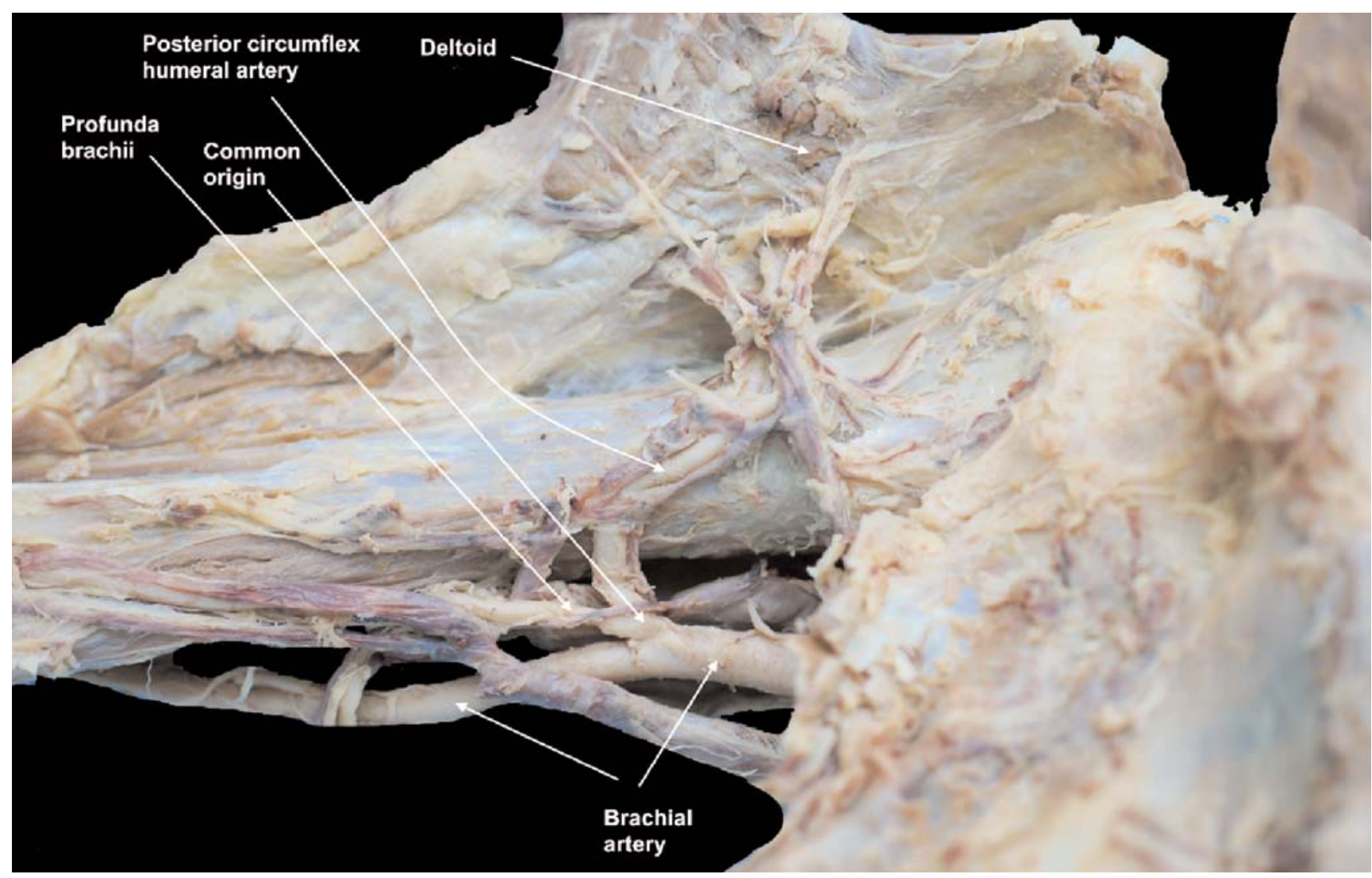

Figure 5. Posterior view of the left shoulder showing the common origin of the posterior circumflex humeral artery with the profunda brachii arising from the brachial artery.

mens $(12.1 \%)$, the subscapular artery in $9(9.3 \%)$, the profunda brachii in $4(2.1 \%)$, and the circumflex scapular in 1 $(0.7 \%)$ (Table 2). The posterior circumflex humeral artery arose as a single branch in 91 specimens (65\%), from a common trunk with the anterior circumflex humeral artery in 11 specimens $(7.9 \%)$, with subscapular artery in 30 specimens $(21.4 \%)$, with profunda brachii in 7 specimens $(5 \%)$ and with circumflex scapular artery in 1 specimen $(0.7 \%)$ (Table 3$)$.

The posterior circumflex humeral artery arose from the posterior aspect of the axillary artery in 62 specimens $(44.3 \%)$, posterolateral in 44 specimens (31.4\%), lateral in 27 specimens $(19.3 \%)$, superior in 4 specimens $(2.9 \%)$, inferolateral in 2 specimens $(1.4 \%)$ and posteromedial in 1 specimen $(0.7 \%)$ (Table 4$)$. During its course the posterior circumflex humeral artery gave rise to the anterior circumflex humeral artery in 17 specimens and the profunda brachii in 4 . A significant difference was observed between genders of the profunda brachii or anterior circumflex humeral artery arising as branches of posterior circumflex humeral artery $(\mathrm{p}=0.02)$. No other differences were observed in relation to gender, side, age and posterior circumflex humeral artery variations. The mean diameter of the posterior circumflex humeral artery was $3.98 \pm 1.00 \mathrm{~mm}$, there being no difference between females and males $(p>0.05)$. Furthermore, there was no correlation between age and diameter of the posterior circumflex humeral artery.

Variation of the subscapular artery was found in 62 specimens (44.3\%) (Figures 1, 3, 4 and 6). It arose from the 3 rd part of axillary artery in 127 shoulders $(88.6 \%)$, from the 2 nd part in 12 specimens $(8.6 \%)$, and the profunda brachii in 1 specimen $(0.7 \%)$. In 2 specimens (1.4\%) the subscapular artery gave rise to the posterior circumflex humeral artery. The subscapular artery arises from a common trunk with either the posterior circumflex humeral artery or the anterior circumflex humeral artery in $0.7 \%$ each. The subscapular artery arose as a single branch in 109 specimens $(77.9 \%)$, it had a common origin with the posterior circumflex humeral artery in 27 specimens $(19.3 \%)$, with the anterior circumflex humeral artery, the anterior and posterior circumflex humeral arteries, the posterior circumflex humeral and circumflex scapular arteries, and the posterior circumflex humeral and profunda brachii arteries in 1 specimen $(0.7 \%)$ each (Table 3$)$. 


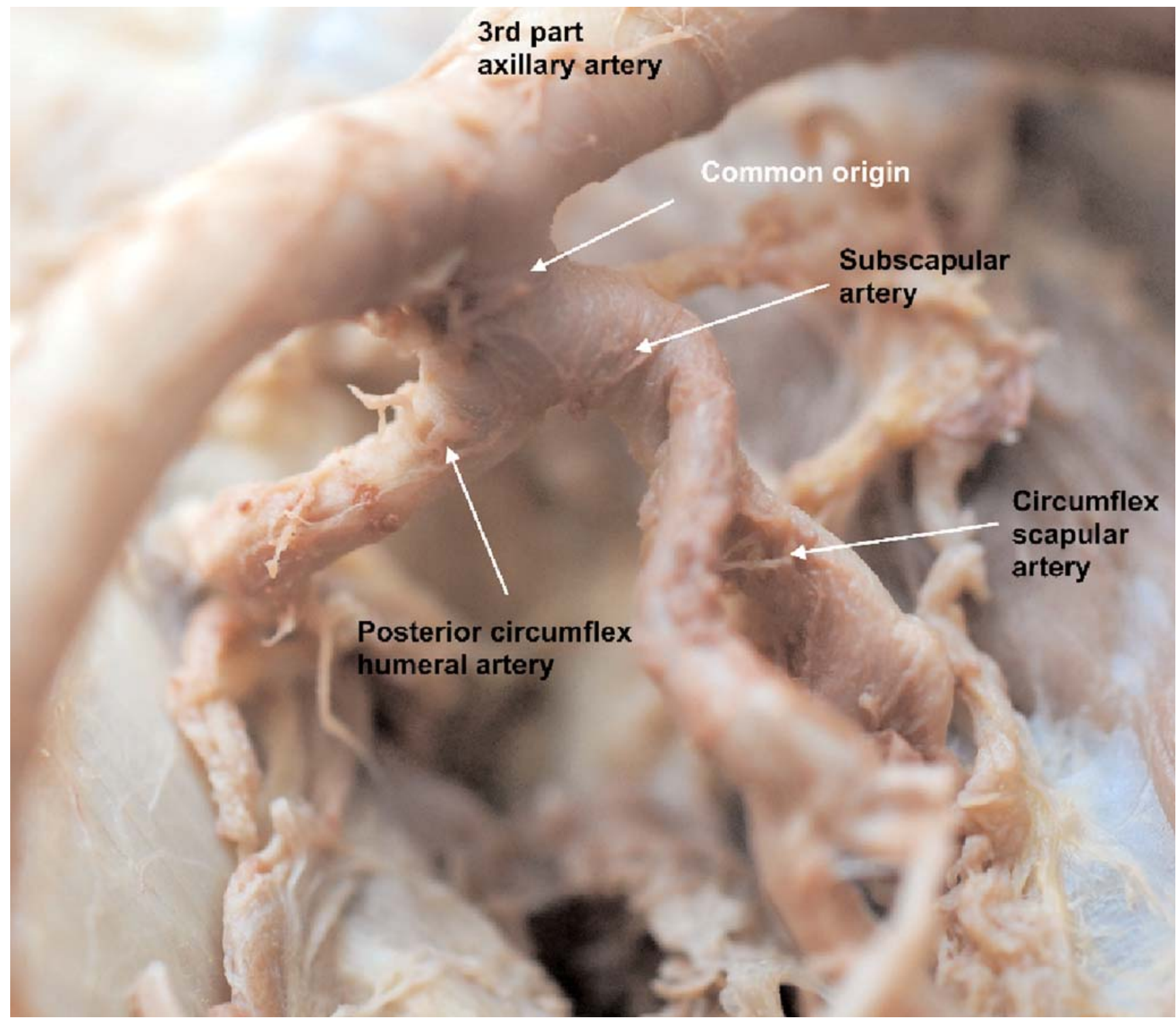

Figure 6. Posteromedial aspect of the right third part of the axillary artery showing the common origin between the subscapular and posterior circumflex humeral arteries.

The subscapular artery arose from the medial aspect of the axillary artery in 97 specimens $(69.3 \%)$, posteromedially in 24 specimens (17.1\%), inferiorly in 12 specimens $(8.6 \%)$ and posteriorly in 7 specimens (5\%) (Table 4). In $92.8 \%$ of specimens, the subscapular artery gave the circumflex scapular artery, and in $7.2 \%$ it gave both the circumflex scapular and posterior circumflex humeral arteries. A variation in the origin of the subscapular artery was observed in 22 males and 40 females. The mean vessel diameter was $5.2 \pm 1.2 \mathrm{~mm}$, with a significant difference being observed between males and females in diameter and origin $(\mathrm{p}<0.05)$. Significant differences were found between vessel diameter and age $(\mathrm{p}<0.05)$.
Considering the above observations together, the most common variants were associated with posterior circumflex humeral artery (48.3\%), followed by the anterior circumflex humeral artery (25\%) and then the subscapular artery $(16.7 \%)$. The anterior circumflex humeral artery arose as a single artery more commonly in females than males $(p<0.05)$, whereas the posterior circumflex humeral and subscapular arteries arose equally frequently in males and females. There was a statistically significant difference $(p<0.05)$ branching pattern according to the origin of the artery between females and males. In females, the anterior circumflex humeral artery was more likely to give the profunda brachii when it originated from the posterior cir- 
cumflex humeral artery. There was a significant association $(p<0.05)$ between the origin of posterior circumflex humeral artery and the branches it subsequently gave. When it originated from the 3rd part of axillary artery, the posterior circumflex humeral artery gave the anterior circumflex humeral artery in $15.1 \%$ of specimens, when it arose from the profunda brachii it gave no branches, when it arose from the circumflex scapular artery it always gave rise to the anterior circumflex humeral artery, and when it originated from the brachial artery it gave the profunda brachii in $11.8 \%$ of specimens.

The posterior circumflex humeral artery gave rise to no branches in $85 \%$ of specimens, but gave rise to the anterior circumflex humeral artery in $12.1 \%$ of specimens and the profunda brachii in $2.9 \%$ of specimens. A significant association $(\mathrm{p}<0.05)$ was observed between the branches given off and gender: in males it was the profunda brachii, while in females it was the anterior circumflex humeral artery.

The site of origin from the 3rd part of the axillary artery in relation to presence or absence of variations are summarized in Table 4. The anterior circumflex humeral artery arose from the lateral aspect of the axillary artery, including posterolateral and anterolateral, aspect of the artery in $91.5 \%$ of specimens. The posterior circumflex humeral artery commonly arose from the 3 rd part from the posterior aspect, including the posterolateral, posteromedial and posterosuperior, in $95 \%$ of specimens. The subscapular artery arose from the medial aspect, including the posteromedial, in $86.4 \%$ of specimens.

\section{Discussion}

The branching pattern of the 3rd part of the axillary artery was studied with respect to its three classical branches, the anterior and posterior circumflex humeral and subscapular arteries, with respect to their origin, variations (if present), diameter and gender. The current study shows a high frequency of variations in the branching pattern, with only $20 \%$ of specimens having the classical branching pattern bilaterally. Of the 140 dissections undertaken and examined variations were noted in $67.9 \%$ of specimens, which were significantly more frequent in females. These observations show that only $32.1 \%$ of specimens followed the description given of textbooks, that the 3rd part of the axillary artery gives rise to three single branches. Consequently, the 'typical' pattern can be considered to less common. Although previous research has reported the frequencies of variation in any one of the three branches, little is known about the incidence of variations. Gaur et al. ${ }^{[2]}$ reported an incidence of variation of $16 \%$ in 25 cadav- ers, while Maheswary Thampi et al. ${ }^{[20]}$ reported an incidence of $40 \%$ in 20 dissected cadavers. The observations of the current study are closer to the findings of Astik and Dave ${ }^{[18]}$ who reported a total incidence of the variant branching pattern of the axillary artery in $62.5 \%$ of their 40 samples; however their findings did not show any gender differences.

Although a high frequency of variations was found, the 3rd part of the axillary artery remained the most frequent site of origin of the anterior circumflex humeral artery $(85.7 \%)$, posterior circumflex humeral artery $(75.7 \%)$ and subscapular artery $(90.7 \%)$. Similarly, in the majority of specimens the anterior circumflex humeral artery $(92.1 \%)$, posterior circumflex humeral artery $(65 \%)$ and subscapular artery $(77.9 \%)$ arose as single branches (Table 3). However, it must be noted that this high incidence did not occur in all specimens. The current high incidence of variation highlights the importance of reporting the incidence of variations in the branching pattern and not merely describe the variation of branches, in order to determine the true incidence of variation in the population.

The anterior circumflex humeral artery arose from the 3rd part of the axillary artery (85.7\%), posterior circumflex humeral artery $(10.7 \%)$, profunda brachii $(2.9 \%)$ and the 1 st part of the axillary artery $(0.7 \%)$ (Table 2$)$. In the majority of specimens $(92.1 \%)$ it arose as a single branch and only in $7.9 \%$ as a common trunk with the posterior circumflex humeral artery (Table 3). Fontes et al. ${ }^{[27]}$ reported that the anterior circumflex humeral artery originated as a single branch in $62.5 \%$ of their sample, from the profunda brachii in $4.2 \%,{ }^{[27]}$ which is a similar to the current study. Both Fontes et al. ${ }^{[27]}$ and Maheswary Thampi et al. ${ }^{[2]}$ reported that it arose from the subscapular artery in $8.3 \%$ of specimens, a variation not observed in the present study. Interestingly, double anterior circumflex humeral arteries have been reported ${ }^{[24,27]}$ and even its absence. ${ }^{[27]}$ The anterior circumflex humeral artery arose from a common trunk in $7.9 \%$ of specimens, agreeing with the $8.3 \%$ reported by Fontes et al. ${ }^{[27]}$ and the $10 \%$ reported by Maheswary Thampi et al. ${ }^{[20]}$

The posterior circumflex humeral artery arose from the third part of the axillary artery in $75.7 \%$ (Table 3), which is in agreement with the $77.1 \%$ reported by Olinger and Benninger. ${ }^{[2]]}$ The second most frequent origin was from the brachial artery (14.2\%), followed by the profunda brachii $(2.9 \%)$, which is lower than the $8.4 \%$ reported by Olinger and Benninger. ${ }^{[28]}$ The origin of the posterior circumflex humeral artery (as a single branch) from the circumflex scapular artery that was observed in the present 
study in $0.7 \%$ has not been reported in previous studies. The $6.4 \%$ arising from the subscapular artery is lower than previous reports: $12 \%$ in Olinger and Benninger, ${ }^{[28]}$ $30 \%$ Kanaka et al. ${ }^{[19]}$ and $54.2 \%$ in Fontes et al. ${ }^{[27]}$ Furthermore, its origin from profunda brachii in $2.9 \%$ of specimens is lower than the $4.2 \%$ of Fontes et al. ${ }^{[27]}$ and the $8.4 \%$ of Olinger and Benninger. ${ }^{[28]}$ Moreover, one study ${ }^{[28]}$ report an origin from the lateral thoracic artery in $1.2 \%$ of their sample: this was not observed in the current study. Although the literature reports the posterior circumflex humeral artery arising as a common trunk with other arteries, ${ }^{[0,18,29]}$ these are case reports, therefore it is not possible to make comparisons with the current study.

Subscapular artery originated form the 3rd part of the axillary artery in $90.7 \%$ of specimens in the current study (Table 2), Olinger and Benninger ${ }^{[28]}$ reported $78.3 \%$. Its origin from profunda brachii has not been previously in the literature. The subscapular artery arising from the $2 \mathrm{nd}$ part of the axillary artery has been reported by Maheswary Thampi et al. ${ }^{[20]}$ in $10 \%$ of specimens and by Gaur et al. ${ }^{[24]}$ in $4 \%$. The current study observed it originated from the 1st first part of the axillary artery in $8.5 \%$ of specimens. Moreover, one study ${ }^{[28]}$ describe the origin of subscapular artery from the lateral thoracic artery in 5.4\%.

The current study is the first to examine variations in males and females separately. We found that significantly more variations were present in females, implying that this should be taken into consideration during medical procedures. Significant differences between genders were found in relation to the origin of the anterior circumflex humeral artery, branches of posterior circumflex humeral artery and the diameter of subscapular artery. Significant differences were also observed when comparing anterior circumflex humeral artery diameter between different age groups, anterior circumflex humeral artery origin and diameter, subscapular artery origin and diameter and age groups. Despite differences in anterior circumflex humeral artery diameter between age groups, no correlation was found between age and the diameter of the artery.

Variations in the origin of vessels from the 3rd part of the axillary artery might be explained by one of three previously suggested developmental theories. ${ }^{[5,30-34]}$ Aharinejad et al. ${ }^{[30]}$ have suggested that variation may be due to remodeling of the complex primitive vascular networks. A second theory, the sprouting theory, suggests that arteries of the upper limb sprout from the axial artery. ${ }^{[0,31,3,3,34]}$ While the third theory postulates that a proximodistal differentiation of the initial capillary plexus, together with a posterior-toanterior polarity, is responsible for development of the arterial pattern of the upper limb. The maintenance, enlargement and differentiation of particular capillary networks and the regression of others leads to the development of the vascular pattern, including its variation. ${ }^{[5]}$

The major strength of the present study is its sample size. All previously published literature had smaller sample sizes, and as such mainly provided information about specific branches of the axillary artery or specific aspects of these branches. Therefore, the literature provides a scattered approach to variations and is not a true representative of the population. In contrast the current study focused on all three branches of the axillary artery describing a range of variables: it therefore provides a more holistic picture of the characteristics of the branches of the 3rd part of the axillary artery. Another further advantage of the current study is that it used gross dissection to examine each artery and its branching pattern. Combined with the large sample size this limits the risk of selection bias.

Despite the advantages of this study, there were some limitations. Even though a variety of aspects of the branching patterns are presented, there is no consideration of the relationship of the arteries to adjacent structures, such as nerves. Such variations have been described elsewhere and are of clinical importance, as they may result in compression of the arteries. ${ }^{[13]}$ Furthermore, the variations described do not include the origin of additional branches from the 3rd part of the axillary artery. Consequently, some variations might not have been identified, so that the frequency of variations may be higher.

The variations in the origin and course of third part of axillary artery are clinically important in diagnostic vascular procedures and surgical treatment. Treatment of chronic shoulder dislocation using axillary approach may injure axillary and brachial arteries. ${ }^{[35]}$ Such variation should be considered during pectoralis major myocutaneous graft, mastectomy or in cardiac bypass surgery for grafting: These variations can be diagnosed and rolled out using doppler study or angiography. It has been also reported that arterial variations can be used to confirm and explain the causes of the upper limb pathologies including peripheral vascular diseases and treatments of axillary artery thrombosis. ${ }^{[36]}$

\section{Conclusion}

This study found a high frequency of variations in the branching pattern whereas when each branch was considered separately a high frequency of "normal" anatomy was found. These results indicate that it is common for one individual to have a variation, but it is uncommon for variations to exist in multiple arteries. Furthermore, this study 
was the first to provide information concerning the relation of variables such as sex, age and side with the presence of variations. Among them, sex was found to be a significant variable influencing the presence of variations in the branching pattern of the third part of the axillary artery. As the topographic anatomy of vascular variations is of major clinical importance, further research should focus on documenting the course of the arteries in relation to adjacent structures.

\section{Acknowledgments}

The authors would like to express their sincere gratitude to the donors and their families. Special thanks to Anatomy at University of Zawia and Centre for Anatomy and Human Identification, University of Dundee.

\section{Conflict of Interest}

None.

\section{Author Contributions}

AMA: collection, processing of material data analysis and interpretation, writing manuscript; TA: data analysis and interpretation, writing manuscript; RS: Proofreading the manuscript.

\section{Ethics Approval}

The specimens were obtained from Centre for Anatomy and Human Identification, which is regulated by the Anatomy Scotland Act (2006).

\section{Funding}

None.

\section{References}

1. Johnson D, Ellis H, Collins P, editors. Pectoral girdle and upper limb. In: Standring S, editor. Gray's anatomy: the anatomical basis of clinical practice. 29th ed. Edinburgh: Elsevier Churchill Livingstone; 2005. p. 799-942.

2. Drake RL, Vogl AW, Mitchell AWM, Gray's anatomy for students. 3rd ed. Philadelphia (PA): Churchill Livingstone Elsevier; 2015. p. 719-819.

3. Moore KL, Dalley AF, Agur AMR. Clinically oriented anatomy. 8th ed. Philadelphia (PA): Wolters Kluwer; 2018. p. 483-91.

4. Maslarski I. The artery blood supply variant of the upper limb. Clujul Med 2015;88:545-9.

5. Rodríguez-Niedenführ M, Burton GJ, Deu J, Sañudo JR. Development of the arterial pattern in the upper limb of staged human embryos: normal development and anatomic variations. J Anat 2001;199:407-17.

6. Çavdar S, Zeybek A, Bayramiçli M. Rare variation of the axillary artery. Clin Anat 2000;13:66-8.

7. Shantakumar SR, Rao KM. Variant branching pattern of axillary artery: a case report. Case Rep Vasc Med 2012;2012:976968.
8. Swamy RS, Rao MKG, Kumar N, Sirasanagandla SR, Nelluri VM. Unusual branching pattern of axillary artery associated with the high origin of ulnar artery. Ann Med Health Sci Res 2013;265-7.

9. Naveen K, Jyothsna P, Satheesha BN, Mohandas Rao KG, Ravindra S, Deepthinath R, Surekha SD. Variant origin of an arterial trunk from axillary artery continuing as profunda brachii artery-a unique arterial variation in the axilla and its clinical implications. Ethiopian Journal of Health Sciences 2014;24:93-6.

10. Aastha, Jain A, Kumar MS. An unusual variation of axillary artery: a case report. J Clin Diagn Res 2015;9:AD05-7.

11. Rao TR, Shetty P, Suresh R. Abnormal branching pattern of the axillary artery and its clinical significance. International Journal of Morphology 2008;26:389-92.

12. Satyanarayana N, Arul moli R, Sunitha P, Chandralekha G, Ravindranath G, Jayasree N, Srilahari N. Variation in the branching pattern of right axillary artery in a malaysian adult male cadaver-a case report. IOSR Journal of Pharmacy 2012;2:19-22.

13. Singh R. Abnormal origin of posterior circumflex humeral artery and subscapular artery: case report and review of the literature. J Vasc Bras 2017;16:248-51.

14. Özgür M, Gölpınar M, Arifoğlu Y, Çavdar S. High origin of radial artery from the axillary artery: case report. Artery Research 2017;17: $39-42$.

15. Olinger A. Upper limb arteries. In: Tubbs RS, Shoja MM, Loukas M, editors. Bergman's comprehensive encyclopedia of human anatomic variation. Hoboken (NJ): John Wiley \& Sons; 2016. p. 583- 618.

16. Venieratos D, Lolis ED. Abnormal ramification of the axillary artery: sub-scapular common trunk. Morphologie 2001;85:23-4.

17. George BM, Nayak S, Kumar P. Clinically significant neurovascular variations in the axilla and the arm-a case report. Neuroanatomy 2007;6:36-8.

18. Astik R, Dave U. Variations in branching pattern of the axillary artery: a study in 40 human cadavers. Journal Vascular Brasileiro 2012;11:12 7.

19. Kanaka S, Eluru RT, Basha MA, Somasekhar R, Kanchanalatha G, Haniman KS. Frequency of variations in axillary artery branches and its surgical importance. International Journal of Scientific Study 2015; $3: 1-4$.

20. Maheswary Thampi S, Vijayamma KN, Prasad Kumar R. Axillary artery - a study on branching pattern and variations. Journal of Evidence Based Medicine and Healthcare 2017;4:261924.

21. Konarik M, Musil V, Baca V, Kachlik D. Upper limb principal arteries variations: a cadaveric study with terminological implication. Bosn J Basic Med Sci 2020;20:502-13.

22. Miguel-Pérez M. Anomalous origin and course of the posterior circumflex humeral artery: clinical interest in shoulder arthroscopy and open procedures. Journal of Orthopedics \& Bone Disorders 2018;2: 000153.

23. Siri AM, Ravikumar V, Gowd HS, Acharya A. Anomalous Branching pattern of the 3rd part of axillary artery. International Journal of Contemporary Medicine 2013;1:68-70.

24. Gaur S, Katariya SK, Vaishnani H, Wani IN, Bondre KV, Shah GV. A cadaveric study of branching pattern of the axillary artery. International Journal of Biological and Medical Research 2012;3: 1388-91.

25. VijayaBhaskar P, Ritesh R, Shankar PR. Anomalous branching of the axillary artery: a case report. Kathmandu Univ Med J (KUMJ) 2006;4: $517-9$. 
26. Pandey SK, Shukla VK. Anatomical variations of the cords of brachial plexus and the median nerve. Clin Anat 2007;20:150-6.

27. Fontes EB, Precht BLC, Andrade RCL, Fernandes RMP, Cisne R. Output relations of humeral circumflex arteries and its variations. International Journal of Morphology 2015;33:1171-5.

28. Olinger A, Benninger B. Branching patterns of the lateral thoracic, subscapular, and posterior circumflex humeral arteries and their relationship to the posterior cord of the brachial plexus. Clin Anat 2010;23:407-12.

29. Banerjee A, Kumari C, Jhajhria SK. Variation in the branching pattern of third part of axillary artery - a case report. J Clin Diagn Res 2017; 11:AD03-4.

30. Aharinejad S, Nourani F, Hollensteiner H. Rare case of high origin of the ulnar artery from the brachial artery. Clin Anat 1997;10:253-8.
31. Jacquemin G, Lemaire V, Medot M, Fissette J. Bilateral case of superficial ulnar artery originating from axillary artery. Surg Radiol Anat 2001;23:139-43.

32. Detton AJ. Grant's dissector. 16th ed. Philadelphia (PA): Wolters Kluwer; 2020. p. 339.

33. Adachi B. Das arteriensystem der Japaner. Vol. 1. Kyoto: Maruzen; 1928. p. 207-85; 350-2.

34. Lippert H, Pabst R. Arterial variations in man. Munich: JF Bergmann-Verlag; 1985. p. 71-3.

35. McGregor AL. A synopsis of surgical anatomy. Bristol: Williams \& Wilkins; 1959. p. 714.

36. Sarkar S, Kundu B, Bose A, Saha PK. Variation of branching pattern of axillary artery. International Journal of Anatomical Variations 2014; $7: 27-9$.
ORCID ID:

A. Alashkham 0000-0003-2959-5039;

T. Almabrouk 0000-0002-2209-4218;

R. Soames 0000-0003-1359-172X
Abduelmenem Alashkham, PhD, MSc, MBBCh

University of Edinburgh, Old Medical School, Doorway 3, Teviot Place, Edinburgh, EH8 9AG, UK

Phone: +441316502924

e-mail: abduelmenem.alashkham@ed.ac.uk

Conflict of interest statement: No conflicts declared.

This is an open access article distributed under the terms of the Creative Commons Attribution-NonCommercial-NoDerivs 4.0 Unported (CC BY-NCND4.0) Licence (http://creativecommons.org/licenses/by-nc-nd/4.0/) which permits unrestricted noncommercial use, distribution, and reproduction in any medium, provided the original work is properly cited. How to cite this article: Alashkham A, Almabrouk T, Soames R. Variations of the branches arising from the third part of the axillary artery: a cadaveric study. Anatomy 2021;15(2):104-115. 